Journal of Mathematics and Statistics 1 (3): 194-202, 2005

ISSN 1549-3644

(C) 2005 Science Publications

\title{
Modeling Traffic Flow and Management at Un-signalized, Signalized and Roundabout Road Intersections
}

\author{
R. Kakooza, L.S. Luboobi and J.Y.T. Mugisha \\ Department of Mathematics, Makerere University, P.O. Box 7062, Kampala, Uganda
}

\begin{abstract}
Traffic congestion continues to hinder economic and social development and also has a negative impact on the environment. A simple mathematical model is used to analyze the different types of road intersections in terms of their Performance in relation to managing traffic congestion and to establish the condition for stability of the road intersections after sufficiently longer periods of time (steady-state). In the analysis, single and double lane un-signalized, signalized and roundabout intersections are evaluated on the basis of their performance (expected number of vehicles and waiting time). Experimental scenarios are carefully designed to analyze the performance of the different types of intersections. It is noted that under light traffic, roundabout intersections perform better than unsignalized and signalized in terms of easing congestion. However under heavy traffic, signalized intersection perform better in terms of easing traffic congestion compared to un-signalized and roundabout intersections. It is further noted that for stability of a road intersection, the proportion of the time a road link stopping at an intersection is delayed should not exceed the utilization factor (the ratio of the arrival rate of vehicles to the product of number of service channels and service rate).
\end{abstract}

Key words: Traffic flow, road intersections, waiting time, expected number

\section{INTRODUCTION}

Traffic congestion causes considerable costs due to unproductive time losses, accidents and also has a negative impact on the environment such as air pollution and fuel; and on the quality of life; noise and stress. The economic consequences of traffic congestion are enormous. Due to the ever-increasing need for transportation, there is more and more traffic congestion in developed and developing countries.

A good understanding of vehicular traffic flow is an important challenge for modern societies. It has a direct impact on our quality of life since most people experience the daily inconvenience of traffic pollution. Traffic implies considerable costs for the community and a great deal of effort is devoted in every large city to reduce the trouble caused by an excess of cars. The presence of traffic congestion costs a lot of money each year in form of lost productivity. In the year 2000, it was estimated that drivers in 75 of the largest metropolitan areas of the world spent more than sixtyeight billion dollars in person hours of lost time and wasted fuel ${ }^{[1]}$. In the United States of America the cost of congestion is estimated to be 67 billion dollars each year $^{[2]}$. This includes 3.6 billion dollars in additional travel time and 5.7 billion gallons of fuel wasted while sitting in traffic.

The Federal Highway Administration spends over 1 billion dollar on traffic mitigation and air quality improvement. Traffic delays result in increased air pollution, thus contributing towards the deterioration of the health and welfare of the citizens. Many of these areas have attempted to alleviate the problems of traffic congestion, by planning and implementing various traffic demand management techniques. In spite of the implementation of these initiatives, such as staggered work hours and flextime, the level and time period of congestion in most major urban areas is increasing ${ }^{[1]}$. In many areas, congestion is no longer limited to two peak hours in a day. It is often extended to two or three hours in the morning, afternoon and evening ${ }^{[3]}$.

Although the problems of traffic congestion are enormous, a complete satisfactory scientific understanding of the phenomena of traffic congestion is still lacking. A reliable scientific description is crucial to study several scenarios and to take a good decision. Depending on the questions to be answered different methodologies can be considered.

For global planning, a description of the traffic flow in terms of a graph, together with standard optimization techniques gives an adequate prediction of the traffic behavior ${ }^{[4]}$. The intersections being the vertices of the graph representing the city and the connecting road segments as the edges. However there is need to identify which intersection design fits a particular location in the transportation network through use of a mathematical model. The first attempts to model traffic flow as a physical process, in terms of equations dates back to the $1960 \mathrm{~s}^{[5]}$. Since then many techniques have been developed, using both continuous (fluid-like) approach and description based on the dynamics of individual cars ${ }^{[6,7]}$.

The problems of a traffic system (such as queues, accidents, pollutant emissions) first appear at

Corresponding Author: J.Y.T. Mugisha, Department of Mathematics, Makerere University, P.O. Box 7062, Kampala, Uganda 
intersections. The meeting point of two or more roads is the focus of conflict since the beginnings of regular traffic $^{[8]}$. At these meeting points (junctions or road intersections) traffic has to be controlled. An early solution was to build up traffic signs separating major and minor roads, but with the growth of motorization and the increase in the number of vehicle users, the traffic exceeded the capacity of the sign-controlled junctions. Thus, a more effective control turned out to be required.

The types of road intersection analyzed include: Un-signalized, Signalized and Roundabout intersections. At road intersections, traffic lights, stop or priority signs are used to constrain car interactions. In case of road intersections controlled by stop signs a waiting time is imposed on cars. In case of Signalized (traffic lights) controlled road intersections, the states (green or red) indicating the permission to go into the intersection (green) or the need to stop before the junction (red) are used. Roundabout on the other hand accommodates traffic flow in one direction around a central island and gives priority to the circulating flow $^{[9]}$. Roundabouts operate on the principle of the mandatory "give-way" rule at all circular intersections, which requires entering traffic to give way, or yield to circulating traffic. This rule prevents circular intersections from locking up, by not allowing vehicles to enter the intersection until there are sufficient gaps in the circulating traffic ${ }^{[10]}$.

In order to model traffic flow at road intersections an understanding of delays is very vital. Queuing delays can be considered as those delays resulting from the presence of other vehicles ${ }^{[11]}$. Traffic at road intersections can be divided into a number of separately identifiable road links each with a capacity and demand flow (approach volume). At peak times the traffic demand on a given road link may approach or even exceed the capacity available to it, whereas in off peak periods there is usually a substantial margin of space capacity. In the morning and evening rush hours, city streets and roads intersection are often jammed with vehicles making it difficult for city resident to get to their places of work in time.

For purposes of testing the validity of the model, data from studies carried out in Kampala City is used. Kampala City has expanded outwards from the Central division along major roads leading to Jinja, Entebbe, Masaka and Luweero. The large parts of the city have developed in an unplanned manner, particularly in the former central Kampala, newly developed in expansion areas and infill situations where settlements have taken place between the planned areas. The development beyond Kampala urban boundaries has been more significant. This is attributed to lack of institutional and regulatory constraints on development in urban areas.

\section{THE MODEL}

We construct a mathematical model to study the impact of varying the parameters of the model on both the expected number and waiting time of vehicles at road intersections.

Description of the model: In the model we refer to a collection of parallel lanes of a road link stopping at a road intersection as a system. The state of the system is looked at as a collection of variables necessary to describe the status of the system. The notation by Kendall $^{[12]}$ is used to denote the queuing system while formulating the model. The $M / M / s$ queuing system used considers s service channels having both inter-arrival and service time distributions independent and identically distributed. In other words the model $\mathrm{M} / \mathrm{M} / \mathrm{s}$ considers a Poisson process with parameter $\lambda$ (arrival rate of vehicles) as its input process and has, for each of the $s$ service channels, independent and identically distributed exponential service time distribution with mean rate $\mu$. The space occupied by a single vehicle on the road link stopping at a road intersection is referred to as a service channel. By service we refer to the act of traveling until the end of the road intersection is reached.

Variables and parameters: The following is a summary of the variables and parameters used in the model:

$W_{Q}: \quad$ the average time that vehicles wait in the queue.

$n: \quad$ the number of vehicles in the queue at time $t$.

$\lambda$ : the average number of vehicles arriving at an intersection per unit time

$s: \quad$ the number of service channels of roads stopping at a road intersection.

$\mu_{0}: \quad$ denotes the service rate with no delays

$\mu_{1}$ : denotes the service rate when interrupted by delays

$f: \quad$ the rate of occurrence of delays

$r$ : the rate of disappearance or clearance of the delays

Let $X(t)$ be the number of vehicles in the system at time $t$ and $Y(t)$ be the status of the system. We define the stochastic process $[X(t), Y(t)]$ to describe the state of the system at time $t$. If at time $t$ the system is delayed then $Y(t)$ is equal to $D$ (delay) otherwise $Y(t)$ is $N$ (normal). The system is said to be in the state $(n, D)$ if there are $\mathrm{n}$ vehicles in the system which is delayed while the system is said to be in state $(n, N)$ if there are $\mathrm{n}$ vehicles in a system which is functioning normally.

Denote the steady-state probability of the system being in state $(n, D)$ by $\pi_{n, D}$ and the steady-state probability of the system in state $(n, N)$ by $\pi_{n, N}$. Let $\mu_{n+1}$ be the service rate when the number of vehicles in the system is $n+1,\left(\lambda_{n}+\mu_{n}\right)$ the sum of the arrival and 
service rates when the number of vehicles in the system is $\mathrm{n}$ and $\lambda_{n-1}$ arrival rate when the number in the system is $n-1$. Also let $f$ and $r$ the rate of occurrence of delays and the rate of disappearance or clearance of the delays to and from states $D$ and $N$ respectively. The transition state diagram is given in Fig. 1.

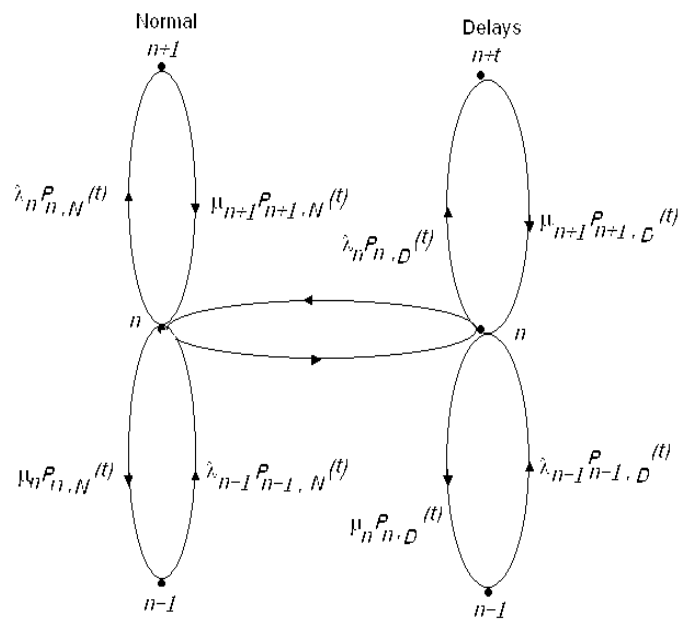

Fig. 1: State transition "forces" diagram for queue with delays

Then the total rate of change in the probabilities of the system $P_{n, N}(t)$ and $P_{n, D}(t)$, that the system is in state $n$ at time $t$ are:

$P_{n, D}^{\prime}(t)=-\left(\lambda_{n}+\mu_{n}+r\right) P_{n, D}(t)+\mu_{n+1} P_{n+1, D}(t)+\lambda_{n-1}$ $P_{n-1, D}(t)+f P_{n, N}(t)$

$P_{n, D}^{\prime}(t)=-\left(\lambda_{n}+\mu_{n}+f\right) P_{n, N}(t)+\mu_{n+1} P_{n+1, N}(t)+\lambda_{n-1}$ $P_{n-1, N}(t)+r P_{n, D}(t)$

For stability (equilibrium) the intensity of leaving state $n$ must be equal to the intensity of moving out of state $n$ (leaving pressure $=$ entering pressure) and in this case we have:

$\left(\lambda_{n}+\mu_{n}+r\right) P_{n, D}(t)=\mu_{n+1} P_{n+1, D}(t)$

$+\lambda_{n-l} P_{n-1, D}(t)+f P_{n, N}(t)$

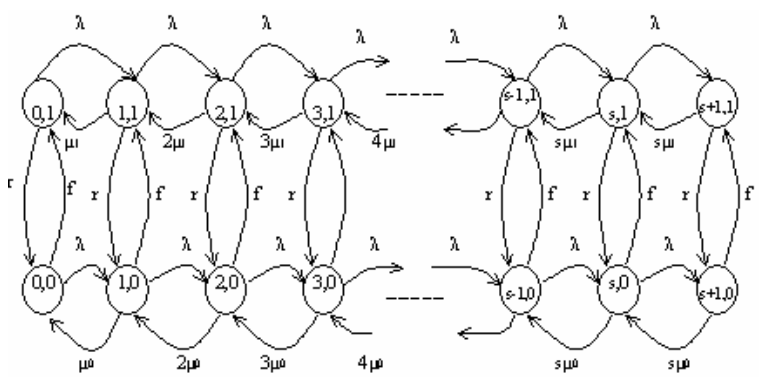

Fig. 2: State transition rate diagram for an M/M/s model interrupted by delays $\left(\lambda_{n}+\mu_{n}+f\right) P_{n, N}(t)=\mu_{n+1} P_{n+1, N}(t)$

$+\lambda_{n-I} P_{n-1, N}(t)+r P_{n, D}(t)$

Equations (1) and (2) can now be applied to an $M / M / s$ queuing system with delays whose transition state diagram is given in Fig. 2. From Fig. 2 we deduce the following:

i. The arrival rate in the entire system is independent of the number of vehicles at the road intersection at the instant of arrival.

ii. If the number of vehicles in the system is less than the number of service channels then the number of vehicles leaving the road intersection for a state with no interruption is given by $\mu_{n}=n \mu_{0}$ and $\mu_{n+1}=(n+1) \mu_{0}$; and for a state interrupted by delays is given by $\mu_{n}=n \mu_{1}$ and $\mu_{n+1}=(n+1) \mu_{1}$.

iii. If the number of vehicles in the system is greater than the number of service channels then the number of vehicles leaving the road intersection for a state with no interruption is given by $\mu_{n}=\mu_{n+1}=$ $\mu_{n-l}=s \mu_{0 ;}$ and for a state interrupted by delays is given by $\mu_{n}=\mu_{n+1}=\mu_{n-1}=s \mu_{1}$.

Then using equations (1) and (2) the steady-state equations for a system interrupted by delays are given by:

Case 1: if the number of vehicles in the system is less than the number of service channels then:

$\left(\lambda+n \mu_{1}+r\right) \pi_{n, D}(t)=(n+1) \mu_{1} \pi_{n+1, D}(t)$

$+\lambda \pi_{n-1, D}(t)+f \pi_{n, N}(t)$

$\left(\lambda+n \mu_{0}+f\right) \pi_{n, N}(t)=(n+1) \mu_{0} \pi_{n+1, N}(t)$

$+\lambda \pi_{n-1, N}(t)+r \pi_{n, D}(t)$

Case 2: if the number of vehicles in the system is greater than the number of service channels then

$\left(\lambda+s \mu_{1}+r\right) \pi_{n, D}(t)=s \mu_{1} \pi_{n+1, D}(t)$

$+\lambda \pi_{n-1, D}(t)+f \pi_{n, N}(t)$

$\left(\lambda+s \mu_{0}+f\right) \pi_{n, N}(t)=s \mu_{0} \pi_{n+1, N}(t)$

$+\lambda \pi_{n-1, N}(t)+r \pi_{n, D}(t)$

With boundary equations:

$(\lambda+r) \pi_{0, D}(t)=\mu_{1} \pi_{l, D}(t)+f \pi_{0, N}(t)$

$(\lambda+f) \pi_{0, N}(t)=\mu_{0} \pi_{l, N}(t)+r \pi_{0, D}(t)$

Qualitative analysis of an $M / M / s$ queue interrupted by delays: During a delay all service channels of a road link stopping at an intersection are closed to traffic. In this case we set $\mu_{1}=0$. The system of equations (3-8) reduce to: 
For $n<s$,

$(\lambda+r) \pi_{n, D}(t)=\lambda \pi_{n-1, D}(t)+f \pi_{n, N}(t)$

$\left(\lambda+n \mu_{0}+f\right) \pi_{n, N}(t)=(n+1) \mu_{0} \pi_{n+1, N}(t)$

$+\lambda \pi_{n-1, N}(t)+r \pi_{n, D}(t)$

For $n \geq s$,

$(\lambda+r) \pi_{n, D}(t)=\lambda \pi_{n-1, D}(t)+f \pi_{n, N}(t)$

$\left(\lambda+s \mu_{0}+f\right) \pi_{n, N}(t)=s \mu_{0} \pi_{n+1, N}(t)$

$+\lambda \pi_{n-1, N}(t)+r \pi_{n, D}(t)$

With boundary equations:

$(\lambda+r) \pi_{0, D}(t)=f \pi_{0, N}(t)$

$(\lambda+f) \pi_{0, N}(t)=\mu_{0} \pi_{1, N}(t)+r \pi_{0, D}(t)$

Let $G_{N}(z)=\sum_{n=0}^{\infty} z^{n} \pi_{n, N}$ and $G_{D}(z)=\sum_{n=0}^{\infty} z^{n} \pi_{n, D}$ for $|z| \leq 1$. Then, the generating function of the number of vehicles in the system in a steady state is given by:

$G(z)=G_{D}(z)+G_{N}(z)$

Multiplying both sides of equations (9) and (11) by $z^{n}$ and summing all over $n$ to infinity yields

$[\lambda(1-z)+r] G_{D}(z)-f G_{N}(z)=0$

Also multiplying both sides of equations (10) and (12) by $z^{n}$ and summing all over $n$ to infinity yields:

$$
\begin{aligned}
& {\left[\lambda(1-\mathrm{z})+\mathrm{f}+\mathrm{s} \mu_{0}\left(1-\frac{1}{\mathrm{z}}\right)\right] \mathrm{G}_{\mathrm{N}}(\mathrm{z})-\mathrm{rG}_{\mathrm{D}}(\mathrm{z})} \\
& =\sum_{\mathrm{n}=0}^{s-1}\left[\left(1-\frac{1}{\mathrm{z}}\right)(\mathrm{s}-\mathrm{n}) \mu_{0} \mathrm{z}^{\mathrm{n}} \pi_{\mathrm{n}, \mathrm{N}}\right]
\end{aligned}
$$

Without loss of generality, the unknown probabilities are $\pi_{0, N}$ and $\pi_{0, D}$. To solve equation (15) and (16), define:

$$
\begin{aligned}
& \underline{g}(z)=\left[\begin{array}{l}
G_{N}(z) \\
G_{D}(z)
\end{array}\right] \\
& A(z)=\left[\begin{array}{cc}
\lambda(1-z)+f+s \mu_{0}\left(1-\frac{1}{z}\right) & -r \\
-f & \lambda(1-z)+r
\end{array}\right] \\
& b_{N}(z)=\sum_{n=0}^{s-1}\left[\left(1-\frac{1}{z}\right)(s-n) \mu_{0} z^{n} \pi_{n, N}\right] \\
& b_{D}(z)=0 \\
& \underline{b}^{\prime}(z)=\left[\begin{array}{l}
b_{N}(z) \\
b_{D}(z)
\end{array}\right]
\end{aligned}
$$

The system of equations (15) and (16) can be rewritten in matrix form as:

$$
A(z) \underline{g}(z)=\underline{b}(z)
$$

Then $g(z)$ can be obtained by inverting matrix $A(z)$. Further manipulations give:

$$
G(z)=\frac{[\lambda(1-z)+r+f] \sum_{n=0}^{s-1}\left[(s-n) \mu_{0} z^{n} \pi_{n, N}\right]}{\lambda^{2} z^{3}-\left(\lambda^{2}+s \lambda \mu_{0}+\lambda f+\lambda r\right) z^{2}+\left(s \lambda \mu_{0}+s \mu_{0} r\right) z}
$$

By definition:

$G(1)=\sum_{n=0}^{\infty} \pi_{n, N}+\sum_{n=0}^{\infty} \pi_{n, D}=1$

Using this fact we have:

$\sum_{n=0}^{s-1}\left[(s-1) \mu_{0} \pi_{n, N}\right]=\frac{s \mu_{0} r-\lambda(r+f)}{r+f}$

For stability of the system, we seek for a probability greater than zero. Thus, a system is stable if

$s \mu_{0} r>\lambda(r+f)$

$\frac{\lambda}{s \mu_{0}}>\frac{r}{r+f}$

$\rho>\frac{r}{r+f}$

We define $\tau=\frac{r}{r+f}$ as the proportion of the time a given road link is delayed. Thus, in order to ease congestion at road intersections, the proportion of the time a road link is delayed should be less than the utilization factor $\rho$ given by $\rho=\frac{\lambda}{s \mu_{0}}$. Using equation (19) and the boundary equations (13) and (14) the values of $\pi_{0, \mathrm{D}}$ and $\pi_{0, \mathrm{~N}}$ are obtained as follows:

$\pi_{0, N}=\frac{s \mu_{0} r-\lambda(r+f)}{r+f}-\frac{1}{s \mu_{0}} \sum_{n=0}^{s-1}\left[(s-n) \mu_{0} \pi_{n, N}\right]$

From the boundary equations (13) and (14) we have

$\pi_{0, D}=\frac{f}{\lambda+r} \pi_{0, N}$

and

$\pi_{1, N}=\frac{1}{\mu_{0}}\left[\lambda+f-\frac{r f}{\lambda+r}\right] \pi_{0, N}$

Knowing $\pi_{0, N}$ and $\pi_{0, D}$, the generating function can be finally obtained by solving (17). Subsequently, the 
expected number of vehicles in the system is achieved by evaluating $\left.G^{\prime}(z)\right|_{z=1}$.

Special cases of the $M / M / s$ queue with delays: Here, we present expressions for expected number and waiting time of vehicles for single and double lane road links stopping at a road intersection interrupted by delays. For each queuing system we assign a particular value of the number of service channels $s$.

a. Delay in single lane system: For an $M / M / 1$ system, Substituting $s=1$ in equation (19) we have:

$$
\pi_{0, N}=\frac{r}{r+f}-\frac{\lambda}{\mu_{0}}
$$

Using the fact that $\pi_{0, D} \in(0,1)$ the stability condition for the $M / M / 1$ queue with delay is obtained as:

$$
\frac{\lambda}{\mu_{0}}>\frac{r}{r+f}
$$

For stability therefore, the traffic intensity $\kappa=\frac{\lambda}{\mu_{0}}$ (in case of a single lane road link) must be less than the proportion of delay time $\tau=\frac{r}{r+f}$.

Substituting $s=1$ in the expression for the generating function given by equation (18) gives:

$$
\mathrm{G}(\mathrm{z})=\frac{[\lambda(1-\mathrm{z})+\mathrm{r}+\mathrm{f}] \mu_{0} \pi_{0, \mathrm{~N}}}{\lambda^{2} \mathrm{z}^{3}-\left(\lambda^{2}+s \lambda \mu_{0}+\lambda \mathrm{f}+\lambda \mathrm{r}\right) \mathrm{z}^{2}+\left(\mathrm{s} \lambda \mu_{0}+s \mu_{0} \mathrm{r}\right) \mathrm{z}}
$$

Combining equations (21) and (22) yields the generating function as

$$
G(z)=\frac{(f+r+\lambda-\lambda z)\left((f+r) \lambda-r \mu_{0}\right)}{(r+f)\left[f \lambda z+(r+\lambda-\lambda z)\left(\lambda z-\mu_{0}\right)\right]}
$$

Evaluating $\left.G^{\prime}(z)\right|_{z=1}$, yields the expression for the expected number of vehicles in the system as:

$$
E(X)=\frac{\lambda\left[(r+f)^{2}+\mu_{0} f\right]}{(r+f)\left[r\left(\mu_{0}-\lambda\right)-\lambda f\right]}
$$

Using Little's formula ${ }^{[13]}$, the average waiting time of vehicles in the system is given by

$$
W=\frac{\left[(r+f)^{2}+\mu_{0} f\right]}{(r+f)\left[r\left(\mu_{0}-\lambda\right)-\lambda f\right]}
$$

b. Delay in double lane system: For an $M / M / 2$ system, Substituting $s=2$ in equation (16) gives

$$
2 \pi_{0, N}+\pi_{1, N}=\frac{2 r}{r+f}-\frac{\lambda}{\mu_{0}}
$$

and from equation (20) we have $\pi_{1, N}=\frac{1}{\mu_{0}}\left[\lambda+f-\frac{r f}{\lambda+r}\right] \pi_{0, N}$

Solving equations (23) and (24) yields $\pi_{0, \mathrm{~N}}$ as

$\pi_{0, N}=\frac{r+\lambda}{r+f} \frac{2 \mu_{0} r-\lambda(r+f)}{2 \mu_{0}(r+\lambda)+\lambda(\lambda+r+f)}$

Using (15) the generating function becomes

$\mathrm{G}(\mathrm{z})=\frac{2 \mu_{0} \mathrm{r}}{\mathrm{r}+\mathrm{f}} \frac{(1-\gamma)(\lambda(1-\mathrm{z})+\mathrm{f}+\mathrm{r})}{\lambda^{2} \mathrm{z}^{2}-\lambda\left(\lambda+2 \mu_{0}+\mathrm{f}+\mathrm{r}\right) \mathrm{z}+2 \mu_{0}(\lambda+\mathrm{r})} \frac{1+\mathrm{z \eta}}{1+\eta}$

Where, $\quad \gamma=\frac{\lambda}{2 \mu_{0}}\left[\frac{r+f}{r}\right]$ and $\eta=\frac{\lambda}{2 \mu_{0}}\left[\frac{r+\lambda+f}{\lambda+r}\right]$

Thus, the expected number of vehicles in this system can be immediately obtained from equation (25) by taking the derivative of $\left.G(z)\right|_{z=1}$. Further manipulations gives:

$E(X)=\frac{2 \lambda \mu_{0}\left[2(\lambda+r)\left(f^{2}+r^{2}+2 \mu_{0} f\right)+f(2 r+\lambda)^{2}\right]}{(f+r)\left(2 r \mu_{0}-f \lambda-r \lambda\right)\left[f \lambda+(r+\lambda)\left(\lambda+2 \mu_{0}\right)\right]}$

Using Little's formula ${ }^{[13]}$, we obtain the average waiting time in the system as:

$W=\frac{2 \mu_{0}\left[2(\lambda+r)\left(f^{2}+r^{2}+2 \mu_{0} f\right)+f(2 r+\lambda)^{2}\right]}{(f+r)\left(2 r \mu_{0}-f \lambda-r \lambda\right)\left[f \lambda+(r+\lambda)\left(\lambda+2 \mu_{0}\right)\right]}$

\section{NUMERICAL SIMULATION OF THE MODEL}

In order to carry out a simulation analysis of the model, parameter values are estimated from data obtained by Kampala Urban Traffic Improvement Plan $^{[14]}$ and from literature.

Parameter values: The parameters that are transformed as inputs for the simulation analysis are as indicated below:

a. Length of the link, $L$ : According to the Journal of Institute of Transport Engineers ${ }^{[15]}$, the average length of occupancy of a vehicle is 17.5 feet, thus the length of an s lane link which can accommodate $C$ vehicles is computed as:

$L=\frac{C \times 17.5}{s}$ feet

$=\frac{0.00525 \times C}{s} \mathrm{~km}$

b. Traffic demands: The number of vehicles arriving at the link per hour: 
$=3600 \lambda($ veh/hour $)$

c. Frequency of delays, $f$ : The rate at which the road intersection is interrupted by delays.

\section{d. Travel speed at full capacity is given by:}

$v=L \times \mu_{0} \times 3600 \mathrm{~km} / \mathrm{hour}$.

e. Delay duration $d=\frac{1}{r}$ seconds, where $\mathrm{r}$ is the rate of disappearance of the delays.

f. To vary the service rate $\mu_{0}$ we use equations (27) and (29).

Combing the two equations and making $\mu 0$ the subject we have:

$\mu_{0}=\frac{v \times s}{18.9 C}$

Since at intersections the vehicle/vehicles at the front leave first or are in the service channels, it is quite reasonable to assume that the number of vehicles in service channels is equal to the number of lanes $(C=$ $s)$. Thus, equation (30) reduces to

$\mu_{0}=0.0529 v$

This implies that the service rate does not depend on the number of vehicles in the system or the number of lanes but on the speed of vehicles. All lanes of an intersection work at the same rate.

g. Delay function: In the simulation analysis it is assumed that all turns corresponding to each link entering an intersection have the same delay. Secondly, the delays associated with intersections for each entering link depends on the characteristics and control policy of that intersection and the volume of traffic on that link. Delays at intersecting links depend not only on the physical characteristics and control policies of intersections, but also on the traffic flows of other links. For the sake of analyzing the impact of varying the parameter of the model on performance measure the following formula by Hedayat and Iravani ${ }^{[16]}$ for delays at road intersections is used.

$d=0.05 \times\left[\frac{n \times(k-1)}{2}\right]$

Where:

$n=$ the number of links ending at the intersection

$k=$ the number of links exiting from the intersection
Thus, $n \times(k-1)$ shows the number of possible turns. The functional form of equation (32) has the following desirable characteristics:

Each link of an intersection, takes into account, the effect of all other links belonging to the same intersection.

Intersections with more links will cause more delay time, assuming the rest of explanatory variables remain the same.

Comparison between un-signalized, signalized and roundabout intersections in terms of performance: Here, we present the results of the operational analysis on the three different types of road intersections. Namely: Un-signalized, Signalized and Roundabout road intersections. Emphasis is given to changes in approach volumes (arrival rates) and delays on the three types of road intersections. The arrival rate is considered to be uniform on all the types of road intersections. Four legged Intersections with single lane road links stopping at them and four legged intersections with double lane roads stopping at them are considered in the comparison. The delay functions used in the calculation of delays at the different types of road intersections are as follows:

a. For un-signalized intersections the delay function given by equation (32) is used in the comparison.

b. For signalized intersections the function used in the comparison is based on Webster's formula ${ }^{[17]}$. According to Webster's model the general equation for replicating the time involved in delays at signalized intersections is given by

$d=\frac{r^{2}}{2 c \times\left(1-\frac{x}{\mu_{0} \times w}\right)}$

Where:

$x=$ traffic volume on entering the link, in vehicles per second.

$\mu_{0}=$ exiting rate of traffic in vehicles per second.

$r=$ red time of the traffic lights in seconds

$c=$ cycle length of the traffic lights in seconds.

$w=$ lane width in meters.

c. For roundabout intersections, the delay function used in the comparison is one given in the NESA manual ${ }^{[18]}$. In the manual the delay function at roundabout intersections is given by

$d=\left(\frac{Q}{S}+0.23 v-5.62-0.12 D+0.000367 v D\right) \lambda$

Where:

$D=$ inscribed circle diameter of the roundabout in meters

$S=0.96 \sqrt{D}+2.03$ meter $/$ second 
Comparison between Un-signalized, Signalized and Roundabout road Intersections (single lane)

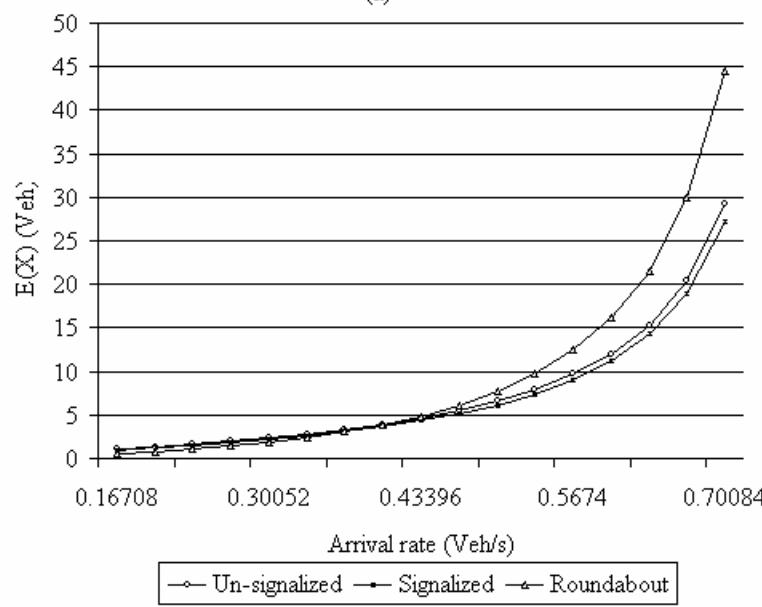

Comparison between Un-signalized, Signalized and Roundabout road Intersections (double lane)

(c)

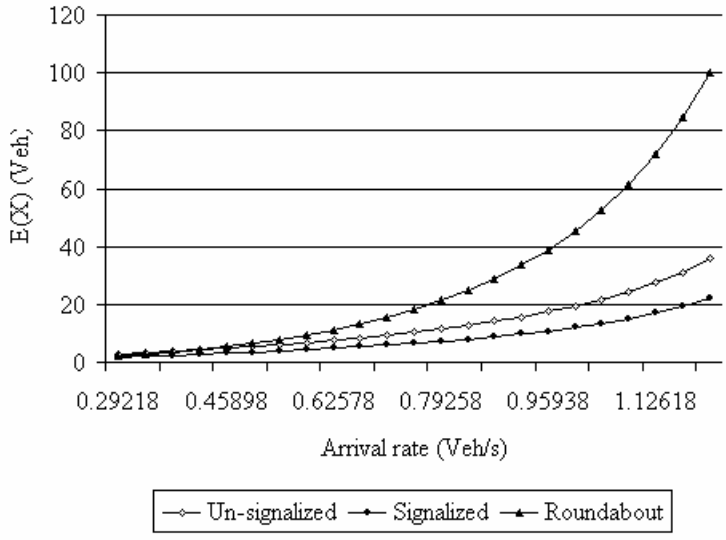

Comparision between Un-signalized, Signalized and Roundabout road intersection (single lane)

(b)

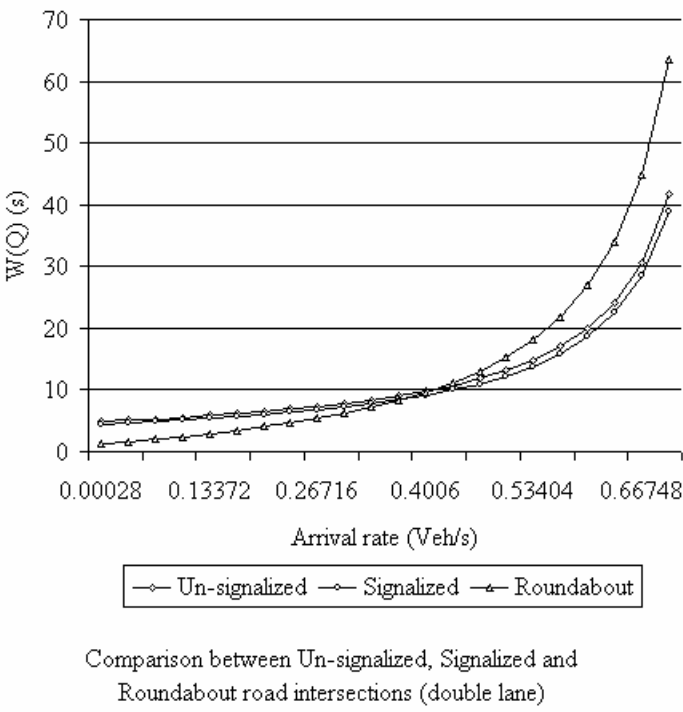

(d)

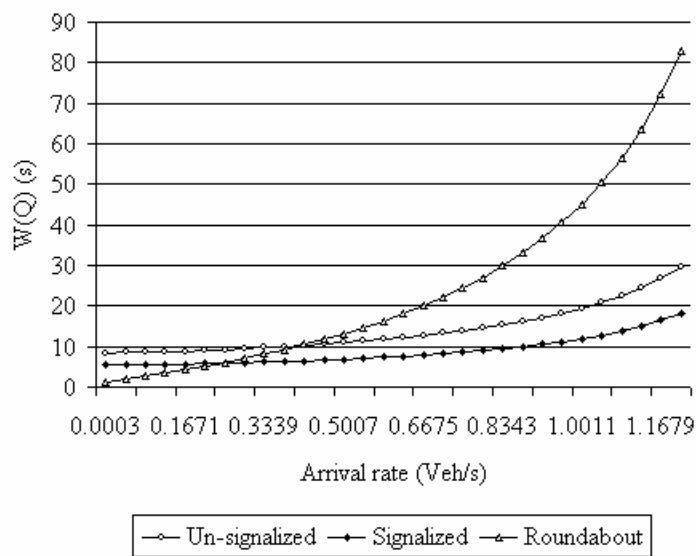

Fig. 3: Comparison between un-signalized, signalized and roundabout intersections in terms of performance

$Q=$ distance traveled on the roundabout

$=\alpha \pi D$ meters

$\alpha=$ proportion of the roundabout traveled

$v=$ the average of the approach speed coded on the entry link

Parameter values for the comparison analysis: The following data values obtained from various studies are used in the comparison.

i. The lane width $\mathrm{w}$ is taken to be 11.67 feet $(3.5$ meters) for all facilities ${ }^{[9]}$.

ii. The diameter of the Roundabout is 100 feet (30 meters) ${ }^{[9]}$.

iii. The red time (r) and the cycle time (c) used in the comparison are 20 and 60 seconds respectively, for a four-legged signalized intersection ${ }^{[9]}$. iv. An average speed of $50 \mathrm{~km} / \mathrm{h}$ is used as the exit speed for all facilities ${ }^{[19]}$.

v. Using the speed given in (iv) above the exiting rate $\mu 0$ can be calculated from equation (31) as:

$\mu_{0}=0.0529 \times 50=2.645 \mathrm{Veh} / \mathrm{s}$

vi. Since the analysis is done during peak periods, traffic volume on entering the link, in vehicles per second $\mathrm{x}$ is assume to be equal to the exiting rate $\mu_{0}$.

vii. In the study, we assume that legs are equally spaced around the Roundabout. For a four leg Roundabout the proportion of the Roundabout traveled $\alpha$ is equal to $0.75^{[18]}$. Figure 3 shows that both the expected number of vehicles and waiting time increase with increase in the arrival rate of 
vehicles (approach volume rate of vehicles). The reason for this is derived from the implication of large values of utilization factor where an increase in the utilization factor increases both the waiting time and expected number of vehicles at road intersections. Since the increase in the arrival rate increases the utilization factor, the expected number of vehicles and waiting time in the system is bound to increase with increase in the arrival rate.

It also noted that at very low values of arrival rate (light traffic), the difference in the expected number of vehicles is not significant Fig. $3 \mathrm{a}$ and $3 \mathrm{c}$. This is so because when traffic is light, all vehicles as soon as they arrive at the intersection be it un-signalized, signalized or roundabout they enter service immediately. As a result differences in performance are not so pronounced. However, when traffic is heavy (high values of arrival rate), the differences in performance become more pronounced with signalized intersection seen to be more superior in terms of expected number compared to un-signalized and roundabout intersections. Un-signalized intersections in turn are seen to perform better than roundabouts in terms expected number when traffic is heavy. These differences in performance may be attributed to differences in geometric factors of the intersections. In case of roundabouts vehicles have to slow down, negotiate the intersection and accelerate back to normal speed. This creates problems if the arrival rate is high. However for signalized and un-signalized intersections, vehicles travel through the intersection straight. Under light traffic, roundabout intersections are seen to perform better than un-signalized and Signalized in terms of waiting time as in Fig. $3 \mathrm{~b}$ and $3 \mathrm{~d}$.

\section{DISCUSSION}

Traffic congestion has continued to be a major hindrance to development in many cities allover the world. It is responsible for the reduction in working hours and deterioration in air quality. A reliable scientific description is crucial to study several scenarios and to take a good decision. The results of this study point out the effectiveness of the different types of road intersections in the bead to ease congestion.

The condition on the parameters $\rho$ (the ratio of the arrival rate of vehicles to the product of number of service channels and service rate) and $\tau$ (the proportion of the time a road link stopping at an intersection is delayed) is used to check the stability (avoiding an ever-increasing back-log of vehicles) of a road intersection. Thus, in order to reduce congestion at road intersection, the proportion of the time a road link stopping at an intersection is delayed $\tau$ should not exceed the utilization factor $\rho$.
The results of the simulation analysis indicate that under heavy traffic, signalized intersections are seen to perform better compared to un-signalized and roundabout intersections in terms of waiting time (Fig. $3 b$ and $3 d)$. The reason for this is derived from the description of the departure flow at stop and give-way lines. In case of signalized intersections, the departures across the stop lines are clearly non random.

Because of cyclic operations of signalized intersections, traffic from each approach road link is released in dense platoons with a substantial gap in between. In this case therefore, the values of both the expected number of vehicles and waiting time are bound to be lower than those for un-signalized and roundabout intersections where the departures across the give-way lines are approximately random. These results are similar to those given in the Florida Roundabout Design Guide. In the guide it is concluded that the performance of signalized intersections is superior under heavy entering traffic volume, while the roundabout works better under light entering traffic volume in terms of delays.

Since congestion is as result of heavy traffic, the best option in managing traffic congestion at road intersection would be to replace un-signalized and roundabout intersections with signalized intersections in case such intersections have heavy approaching traffic. This will help to ease the pressure on these intersections and consequently reduce congestion at these intersections.

\section{ACKNOWLEDGEMENTS}

The authors would like to thank Rev. Fr. J. Scalabrini, Chairman Board of Governors Bishop Cipriano Kihangire S.S and NUFU for the financial support.

\section{REFERENCES}

1. Texas Transportation Institute. The 2002 Urban Mobility Report.

2. U.S House Committee on Transportation and Infrastructure (March 26, 2003). Washington D.C.

3. Kerrie, L.S., S.M. Deborah and K.D. Tapan, 2004. A test and validation of traffic performance characteristics of a coordinated traffic signal system. Transportation Research Group, Department of Civil and Environmental Engineering. Wayne State University.

4. Alexandre, D. and C. Bastien, 2003. Parallel simulation of traffic in Geneva using cellular automata. http://www.spiess.ch/emme2/.

5. Prigogine, I. and R. Herman, 1971. Kinetic Theory of Vehicular Traffic. American Elsevier, New York.

6. Wilhelm, L., 1988. Introduction to the Theory of Traffic Flow. Berlin. 
7. Wolf, D.E., M. Schreckenberg and A. Bachem, 1996. Tra_c and granular flow. Proc. Intl. Workshop in Juelich World Scientific, Singapore, pp: $137-149$.

8. Laszlo, S.K., 1998.Survey of capacity and traffic performance at a T-intersection in Trondheim, Norway. Technical University of Budapest Scientific Conference of students.

9. Virginia, P.S. and P.G. Veera, 2004. Design and safety of modern roundabouts. Access Board. Pedestrian Access to Modern Roundabouts, Department of Civil and Environmental Engineering. University of Alabama. Birmingham.

10. Robinson, B.W., 2000. Roundabout, An Informational Guide. Publication FHWA-RD-00067. FHWA, U.S. Department of Transportation.

11. COBA Manual, 2002. Economic Assessment of Road Schemes. Vol. 13: Part 6.

12. Kendall, D.G., 1951. Some problems in the theory of queues. J. Royal Stat. Soc., Series B, 13: $151-185$.
13. Little, J.D.C., 1961. A proof for the queuing formula $\mathrm{L}=\lambda \mathrm{W}$. Operations Research, 9: 383-387.

14. Kampala Urban Traffic Improvement Plan (KUTIP), 2004. RITES Ltd., India, In association with Rank Consults (U) Ltd and Kagga \& Partners Ltd., Uganda.

15. ITE., 1994. Operational evidence of changing travel patterns. A case study. ITE Journal.

16. Hedayat, Z.A. and H. Iravani, 1999. Use of intersection delay functions to improve Reliability of Traffic assignment models. Presentation at the Ann. Intl. EMME/2 Conf., Chicago, Illinois.

17. Webster, F.V., 1958. Traffic Signal Setting. Report 39, Road Research Laboratory Crowthorne. Berkshire, England.

18. NESA Manual, 1998. Design Manual for Roads and Bridges. Economic Assessment of Road Schemes in Scotland.

19. Wang, R. and H.J. Ruskin, 2003. Modeling traffic flow at single-lane urban roundabout. Comp. Phys. Commun., 147: 570-576. 